\title{
La tradition de Chicago et l'étude des relations entre les races
}

Jean-Michel Chapoulie

\section{(2) OpenEdition}

1 Journals

Édition électronique

URL : https://journals.openedition.org/remi/1600

DOI : $10.4000 /$ remi. 1600

ISSN : $1777-5418$

Éditeur

Université de Poitiers

\section{Édition imprimée}

Date de publication : 12 décembre 2002

Pagination : 9-24

ISBN : 2-911627-32-6

ISSN : 0765-0752

\section{Référence électronique}

Jean-Michel Chapoulie, «La tradition de Chicago et l'étude des relations entre les races », Revue européenne des migrations internationales [En ligne], vol. 18 - n³/2002, mis en ligne le 09 juin 2006, consulté le 15 avril 2022. URL : http://journals.openedition.org/remi/1600 ; DOI : https://doi.org/ 10.4000/remi. 1600

Ce document a été généré automatiquement le 15 avril 2022.

(c) Université de Poitiers 


\title{
La tradition de Chicago et l'étude des relations entre les races
}

\author{
Jean-Michel Chapoulie
}

En retenant comme thème de cet article la contribution de la tradition de Chicago à l'étude des relations entre les races, je me proposais d'attirer l'attention sur ce qui me semblait à la fois le plus central et le plus élaboré, mais aussi le plus mal connu en France dans les recherches de cette tradition. L'examen montre que les choses ont commencé à changer, puisque certains travaux récents sont consacrés à ce thème. La raison profonde en est à l'évidence que les chercheurs en sciences sociales dont ce n'est pas la spécialité découvrent lentement et péniblement depuis une quinzaine d'années non seulement que la question des relations entre races et cultures concerne directement la France, mais qu'elle l'a concernée depuis un bon siècle sans que les sciences sociales s'en soient souciées. Pour emprunter le vocabulaire de Park, la France se trouve, comme d'ailleurs la plupart des pays où s'est développée une grande industrie, sur une frontière ethnique et raciale. Une remarque de Park dans un essai de 1926 peut suggérer l'actualité qu'ont aujourd'hui ses essais sur les relations entre races et cultures: "Nous avons importé de la main-d'œuvre comme si c'était une simple marchandise, écrit Park, et de temps en temps nous sommes déçus lorsque nous découvrons, comme cela se produit invariablement, que les travailleurs étaient des êtres humains comme nous » (Park, 1926/ 1950 : 150).

2 Puisque les sociologues de la tradition de Chicago ont depuis presque un siècle étudié ces phénomènes, et la conviction est présente que leurs recherches ont abouti à des « résultats » qui méritent encore aujourd'hui d'être connus, je me propose de remplir le rôle d'intermédiaire culturel et d'indiquer ce qui est, selon moi, leur contribution centrale à l'étude des relations entre races et cultures : un cadre de référence dont W. I. Thomas et R. Park ont fourni la formulation première ${ }^{1}$. J'indiquerai plus loin pourquoi on ne peut pas utiliser facilement les présentations de la sociologie de Park et de ses successeurs que l'on trouve chez les spécialistes américains des contacts de races et cultures. J'examinerai ensuite rapidement les relations de ce cadre de référence avec une partie des recherches empiriques ultérieures de deux des « élèves » de Park les plus 
influents, E. Franklin Frazier et Everett Hughes. Un peu à l'arrière-plan, on trouvera des éléments de réflexion sur deux questions au centre de mes recherches sur l'histoire des sciences sociales ${ }^{2}$ : quelle est la nature des accomplissements des sciences sociales? Quelle est la relation entre ces recherches et la société qui les a produites?

Le cadre d'analyse de Park

On ne sera pas surpris que le rôle d'intermédiaire culturel impose d'abord de rappeler le sens d'un mot. Le terme race peut inquiéter certains. Je rappellerai donc que ce terme - ou plutôt le terme anglais correspondant - ne renvoie pas à une définition biologique, mais à une définition strictement sociale : comme le remarquait un élève de Park, Everett Hughes, en 1967 : « La seule chose évidente à quelqu'un de complètement extérieur est que la race dans ce pays est une invention linguistique ; naturellement il y a beaucoup d'histoire derrière celle-ci, comme derrière toute invention linguistique " (Hughes, 1967) ${ }^{3}$. Au moins à partir des années vingt, le terme race renvoie pour Park et ses proches à une définition non pas biologique, mais sociale, et c'est un premier indice $\mathrm{du}$ fait que la réflexion de la sociologie américaine avait déjà un raffinement incomparablement supérieur à la nôtre dans l'appréhension de cet ordre de phénomènes.

4 La raison de ce raffinement n'est pas mystérieuse : la première interrogation de la société américaine sur elle-même, quand naît la sociologie aux États-Unis, autour de 1890, porte sur la coexistence de populations diverses sur le sol des États-Unis. C'est le thème de Hull House Maps and Papers publié par Jane Addams et ses proches en 1895, du Philadelphia Negro de Du Bois (1899), du Paysan Polonais de Thomas et Znaniecki (1918). C'est aussi le thème principal du manuel de Park et Burgess, l'Introduction to the Science of Sociology (1921) auquel je m'arrêterai.

5 La majeure partie de cet ouvrage est consacrée à la présentation d'un cadre conceptuel destiné à l'analyse des contacts entre populations différentes présentes sur un même territoire. Celui-ci repose sur un ensemble de notions - interaction, contrôle social, etc. - trop éloignées de nos terminologies et de nos modes de pensée ordinaires pour qu'il puisse être présenté ici en détail. On peut cependant donner une idée d'ensemble du cadre d'analyse que Park et Burgess offraient aux étudiants en thèse en avançant qu'il s'agit d'une sorte d'abstraction inspirée par la situation historique qui était celle des États-Unis : un vaste territoire où s'était installée une émigration de peuplement ininterrompue sur plus d'un siècle, et sur lequel une organisation politique se mettait lentement en place (Karl, 1983). La concurrence de ces diverses populations en ce qui concerne les emplois et le logement était un phénomène évident pour tout observateur ${ }^{4}$. Le point de vue organisant cette perception est celui des Anglo-saxons protestants, blancs, originaires du Nord ; le groupe politiquement hégémonique.

6 Ni pour Park ni pour ses contemporains, les relations entre ces populations arrivées par vagues successives d'immigration n'étaient caractérisées par une grande stabilité : il leur semblait donc approprié de les considérer comme des phénomènes soumis à des évolutions rapides et de rechercher les régularités dans le déroulement des processus et non dans des états stables et tendant à se répéter. Park et Burgess proposent une classification de ces processus. Le premier d'entre eux, la concurrence (compétition) pour l'occupation d'un territoire, l'accès aux emplois, etc. - relève de ce que Park appelle l'ordre écologique : le domaine des relations qui ne supposent nécessairement ni contact direct ni communication entre les populations concernées. Les trois autres types de processus - conflit, compromis, et assimilation - impliquent au contraire des 
formes de communication et relèvent de ce que Park appelle l'ordre moral ${ }^{5}$. L'étude de la dimension symbolique de ces formes de communication est l'un des principaux centres d'intérêts de Park, qui fut l'un des introducteurs en sociologie de la démarche que nous désignons maintenant par le terme « travail de terrain » (fieldwork).

7 Comme souvent chez Park, la définition de certaines notions n'est qu'esquissée ${ }^{6}$. Il en va ainsi pour la notion d'assimilation. Il faut rappeler - parce que les lecteurs des trente dernières années semblent l'ignorer - que le terme "assimilation » renvoie moins à une idée de spécialiste de sciences sociales qu'au thème d'un des principaux débats publics de l'époque : en 1919, sur "l'américanisation » des émigrants, ou plus généralement sur l'avenir des émigrés européens et asiatiques et après 1920, sur celui des Noirs. Explicitement formulée seulement dans l'article de 1926 cité précédemment (mais sous-entendu dans l'Introduction to the Science of Sociology), une hypothèse sur l'évolution des relations entre ces populations est avancée par Park : cette évolution se conformerait à un «cycle de relations » qui ferait inexorablement passer les relations d'un de ces processus à l'autre.

8 L'une des infortunes dont a souffert la sociologie de Park est cependant l'excessive identification, si ce n'est sa réduction, à cette proposition : c'est en effet à celle-ci que se limite souvent depuis vingt-cinq ans l'exposé de l'approche de Park, ce qui conduit d'ailleurs inévitablement à la déclarer obsolète, non scientifique, etc. ${ }^{7}$. La discussion autour de l'interprétation des essais de Park ne peut évidemment aboutir à aucune conclusion incontestable. Je soutiens seulement ici que ceux qui se sont inspirés des essais de Park dans des recherches empiriques n'ont pas été obnubilé par l'idée de démontrer l'exactitude de cette proposition et qu'une lecture des essais de Park qui n'est pas focalisée autour de l'hypothèse de ce cycle suggère d'autres idées bien plus fécondes pour des recherches empiriques.

9 De proches héritiers intellectuels de Park, comme Hughes ou Frazier, n'attachaient d'ailleurs pas une grande importance à ce cycle ${ }^{8}$. Ni d'ailleurs souvent Park lui-même qui se réfère volontiers au cas des Juifs en Europe. Une remarque dans un manuscrit de Park montre qu'il n'avait pas une vision dogmatique de cette proposition : «Ce cycle, écrit Park, est une hypothèse, et la seule façon acceptable dans tous les cas de travailler avec une hypothèse est de chercher quand et dans quelles circonstances celle-ci n'est pas exacte $»^{9}$. On peut avancer que l'intérêt porté à l'hypothèse d'un " cycle de relation entre les races » est l'une des expressions de la prégnance dans les débats publics aux États-Unis de la question de l'avenir des différentes composantes de la population et de l'état de leurs relations, et spécialement de l'avenir des relations des Noirs avec les autres composantes de la société américaine.

10 La difficulté d'interprétation des recherches américaines sur les relations entre races et cultures dépasse la question de la place à accorder au cycle des relations entre les races. Tout ce qui touche aux relations de race est, au moins depuis 1920, une question brûlante pour les sciences sociales aux États-Unis car directement liée à un débat politico-moral central. En conséquence, l'appréciation des analyses de sciences sociales, contemporaines ou passées, qui relèvent de ce domaine, dépend des jugements constitués sur tel ou tel aspect sensible à ce moment, même si cet aspect n'est pas central pour les analyses considérées. L'appréciation des essais de Park a ainsi souffert de la "découverte» à la fin des années soixante que l'on pouvait aussi analyser l'évolution de la population Noire comme un phénomène $d$ ' "ethnogenèse " (curieusement : car Park était sans doute presque seul de son temps à être attentif à cet 
aspect ${ }^{10}$ ), ainsi que de la découverte que l'héritage culturel africain n'était pas aussi négligeable que l'avaient longtemps affirmé avec Park à peu près tous les chercheurs de sciences sociales. Cette focalisation des appréciations portées sur les analyses sur quelques points sensibles par rapport aux débats du moment des États-Unis rend souvent difficilement utilisables par le lecteur non initié à ces débats les présentations des essais de Park et de ses successeurs par les chercheurs américains ${ }^{11}$. On peut ajouter aussi qu'une partie du cadre de référence proposé par Park a été intégré dans les catégories de base de ce domaine d'études et que ce n'est donc plus directement aux essais de celui-ci qu'est associée, par exemple, l'idée que les relations de races et non les groupes en eux-mêmes sont l'objet d'étude central de ce domaine.

11 J'ai passé jusqu'ici sous silence une propriété de la perspective proposée par Park qui ne frappe plus le lecteur d'aujourd'hui, car elle renvoie au contexte intellectuel de l'époque dans laquelle celle-ci s'est développée, mais qui était excessivement importante aux yeux de Park et de ses premiers lecteurs. Park récuse à peu près toute idée d'inégalité biologique entre les races: il considère les différences entre populations éventuellement observables à un moment donné comme le produit de l'environnement ou des environnements successifs ${ }^{12}$. En cela Park s'oppose aux convictions qui sont encore très répandues dans les sciences sociales au début des années vingt, même si, sous l'influence des anthropologues boasiens, les convictions racistes reculent chez les chercheurs de sciences sociales à partir du début du siècle. Les ouvrages de plusieurs des «élèves » de Park, comme Charles Johnson, E. Franklin Frazier, mais aussi ceux de Clifford Shaw et de Henry McKay sur la délinquance, contiennent ainsi parfois de longues démonstrations du fait que la race ou l'appartenance ethnique n'ont aucune valeur pour l'explication de comportements spécifiques (comme les ruptures familiales ou la délinquance).

12 J'ai fait allusion à deux autres caractéristiques importantes du cadre d'analyse proposé par Park que je rappelle ici seulement pour mémoire. Premièrement, ce ne sont pas les races ou les groupes ethniques en eux-mêmes, mais les relations de race qui sont l'objet central de cette perspective. Deuxièmement, ce n'est pas la régularité de ces relations qui doit être analysée, mais la régularité des évolutions, car la société est en perpétuel devenir. Je développerai un peu plus longuement trois autres propriétés importantes de ce cadre d'analyse.

13 Troisièmement : le cadre d'analyse de Park accorde une attention centrale aux conflits entre groupes. Au moins à la fin de sa vie, ces conflits, y compris les émeutes raciales, sembleront à Park l'un des intermédiaires inévitables aux changements sociaux ${ }^{13}$. La perspective proposée par Park s'oppose sur ce point à celle qui inspire l'immense majorité des recherches sociologiques dans ce domaine après 1945, centrées sur les attitudes et les préjugés dans leurs expressions individuelles ${ }^{14}$. Ces recherches ont porté en conséquence davantage sur les groupes en eux-mêmes que sur les relations, davantage focalisées sur les réactions individuelles que sur les comportements collectifs, moins attentives aux conflits que convaincues que l'amélioration progressive des relations passe par l'atténuation de ceux-ci, et tournées davantage vers la compréhension de l'état des relations de races plutôt que vers l'explicitation des processus de changement.

14 Quatrièmement: Park considérait, même si le point reste à l'arrière-plan dans ses essais, que la base des mouvements de population qui entraîne les contacts entre races et cultures est essentiellement économique : c'est la recherche de travail qui a mis en 
mouvement depuis le début du XIXe siècle une partie des populations du monde. Park utilise l'expression « expansion de l'Europe », empruntée à l'anglais James Bryce, pour désigner ce phénomène de grande ampleur qui se développe depuis plusieurs siècles mais dont il percevait dans les années trente l'accélération, à juste titre comme devait le confirmer la suite. Derrière cette interprétation, d'un des phénomènes majeurs depuis la fin $\mathrm{du}_{\mathrm{XIX}}^{\mathrm{e}}$ siècle, se trouve une réflexion, commune à Park et à une partie des chercheurs de sciences sociales de l'époque, sur l'opposition entre sociétés fondées sur les contacts intimes tels qu'il en existe dans la famille, et sociétés caractérisées par les contacts impersonnels caractéristiques de l'univers des affaires, de la politique et des villes (Park, 1939b in Park, $1950: 116)$. Cette réflexion débouche sur l'attention portée aux contributions respectives de la famille et de diverses institutions - écoles, églises, presse, etc. - à l'adaptation des groupes issus de l'immigration dans la société qui les accueille, et aux différences entre les générations successives issues d'une même immigration.

15 Cinquièmement : si le point de départ est l'exemple historique des États-Unis du début $\mathrm{du} \mathrm{xx}^{\mathrm{e}}$ siècle, la réflexion de Park se situe dans un cadre comparatif que celui-ci n'a cessé d'élargir au fil des années; ce cadre englobe certes les Asiatiques de la côte Pacifique des États-Unis et les groupes ethniques soumis au même processus d'urbanisation que les Noirs ruraux du sud, mais également des exemples connus par Park à l'occasion de ses visites des années 1920-1935 comme l'Inde, la Chine, les Iles Hawaî ${ }^{15}$, l'Afrique du Sud, le Brésil, ainsi que des exemples historiques auxquels Park se réfère fréquemment: les Juifs d'Europe, le mouvement des Nationalités en Europe centrale, la Grèce antique, etc. C'est en s'appuyant sur des comparaisons effectuées dans ce cadre large que Park a avancé ses analyses des conséquences de la différenciation sociale sur les relations entre races, du rôle de la conscience de race, des préjugés de race comme sens de la position relative des groupes, des comportements de ceux qui se trouvent marginaux par rapport aux communautés de race.

Les analyses de Park dans le domaine des contacts entre races et cultures sont incomparablement plus élaborées et subtiles que celle qu'il propose pour d'autres sujets, y compris pour l'étude des villes ${ }^{16}$. C'est en effet dans ce seul domaine que Park (qui était devenu professeur de sociologie à 50 ans) a réalisé directement des recherches participant lui-même à la collecte et à l'interprétation d'un matériel documentaire (Park, 1922). La recherche avait été financée par la Fondation Carnegie, sur les formes d'adaptation de divers types d'immigrés (en collaboration avec W.I. Thomas) ${ }^{17}$, et sur l'émigration asiatique de la Côte Pacifique des États-Unis ${ }^{18}$. On doit y ajouter les travaux, inspirés par Park, de la commission d'enquête consécutive à l'émeute de Chicago de 1919 (dont l'auteur principal fut un élève de Park, Charles Johnson). Park était par ailleurs le seul chercheur de sa génération à posséder une connaissance quasi ethnographique de la condition des Noirs du sud rural ${ }^{19}$. À partir du début du siècle, il n'a cessé de scruter les évolutions des comportements individuels et collectifs de la population Noire, les événements qui la concernent et les réactions suscitées par ces évolutions : soit notamment les tentatives de Booker Washington pour organiser un développement économique et culturel à l'écart des Blancs au début du siècle, la Grande Migration des Noirs ruraux du sud vers les villes du Nord après 1915 ; la Harlem Renaissance des années vingt, etc. Ses essais, mais plus encore ses brouillons et sa correspondance, témoignent de l'acuité de sa perception. 
17 Bien qu'il ne s'agisse pas ici de présenter une appréciation critique du point de vue de Park, il est sans doute utile de rappeler qu'il a laissé de côté une partie de la dimension politique des relations de races : il tend à voir la concurrence entre celles-ci comme un phénomène non organisé et à passer sous silence les actions collectives des Blancs en la matière. Il a également négligé les possibilités d'intervention de l'État fédéral sur les relations de races. On peut mettre ces omissions en relation avec les propriétés de son point de vue que j'avais relevées en commençant : c'est seulement après la Première Guerre mondiale que se développent les interventions de l'État fédéral aux États-Unis dans différents domaines; par ailleurs Park était selon ses termes, un «démocrate jeffersonien" profondément hostile à ces interventions (Matthews, 1977: 183). L'appartenance de Park au groupe anglo-saxon protestant, le groupe de statut le plus élevé, ne le prédisposait peut-être pas non plus à porter attention aux actions concertées du gouvernement fédéral en faveur du maintien en l'état des relations de races.

18 La présentation synthétique que je viens d'en donner risque de suggérer que le cadre d'analyse de Park préexistait aux recherches empiriques, voire que ni celles-ci, ni les évolutions et les événements qu'il observe ne l'auraient modifié. Je laisserai de côté la question des inflexions qu'il apporte à son cadre d'analyse entre le début et la fin de sa carrière et j'examinerai seulement la relation de celui-ci aux recherches ultérieures.

L'héritage de Park

19 Entre 1930 et les années soixante (au moins) de nombreuses recherches empiriques ont adopté le cadre de référence de Park et constituent donc une mise à l'épreuve de celuici. On y trouve les travaux de ceux qui furent à un moment ou un autre ses élèves ou ses proches, comme Charles Johnson, E. Franklin Frazier, Everett Hughes, Bertram Doyle, William O. Brown, Edgar Thompson, Donald Pierson, Andrew Lind, voire Ira de A Reid, pour nommer le troisième grand sociologue Noir de la génération née autour de 1900, ou Romanzo Adams, un diplômé de l'Université de Chicago presque de la génération de Park. On peut aussi mentionner Louis Wirth et Herbert Blumer qui, sans réaliser euxmêmes véritablement de recherches empiriques dans le domaine, ont développé des critiques souvent incisives sur ces sujets et inspiré une partie des travaux des chercheurs de la génération suivante, comme Tamotsu Shibutani et Lewis Killian. Pour montrer le fonctionnement du cadre de référence élaboré par Park, j'examinerai rapidement une petite partie de la contribution des deux élèves de Park qui furent en leur temps les plus influents dans le domaine, E. Franklin Frazier et Everett Hughes ${ }^{20}$.

20 S'il a consacré, à l'inverse de Park, à peu près la totalité de sa carrière à l'étude d'un seul cas, les Noirs américains, E. Franklin Frazier avait acquis en la matière une expérience plus large que quiconque dans sa génération ${ }^{21}$. Peu apprécié des organismes de financement de son temps en raison notamment d'opinions jugées trop « radicales », il a cependant mené à bien un nombre impressionnant de travaux empiriques sur des aspects essentiels de l'existence des Noirs américains. Sa première notoriété fut établie par un ensemble de recherches sur la famille Afro-américaine des États-Unis et sa constitution historique (Frazier, 1932; 1939). Ces analyses, qui contiennent une argumentation massive en faveur d'une interprétation environnementaliste des singularités des comportements familiaux des Noirs américains, ont été longtemps la référence centrale sur le sujet ${ }^{22}$.

21 Après ces premières recherches, Frazier a contribué à un vaste programme d'étude des comportements des jeunes Noirs des années quarante, à côté de Charles Johnson, Lloyd 
Warner et Ira de A Reid (Frazier, 1940), puis, dans un livre plus polémique publié d'abord en français, il a analysé les comportements de sa propre classe, la petitebourgeoisie Noire (Frazier, 1955), avant de donner une nouvelle formulation à la problématique de l'expansion de l'Europe (Frazier, 1957) et d'étudier les églises comme institutions de la communauté Noire américaine ${ }^{23}$.

Les recherches sur ces deux institutions majeures pour l'adaptation des immigrants ruraux, que sont la famille et la religion, témoignent de la relation étroite qui unit la sociologie de Frazier et celle de Park. L'héritage de Park est d'ailleurs revendiqué dans l'essai de Frazier qui constitue son allocution en tant que président de l'Association américaine des sociologues ${ }^{24}$. Cet essai développe une présentation de ce qui constitue l'un des apports de Frazier à l'enrichissement de la perspective de Park : l'attention aux incidences de la structure de classe sur les relations de race et leurs évolutions. Les années vingt avaient été marquées par l'occultation quasi complète de la notion de classe sociale dans la sociologie américaine (Gilkeson, 1995; Chapoulie, 1998). Contrairement à Park qui manifestait une certaine ambivalence à l'égard de la notion qu'il n'utilise d'ailleurs que dans ses essais sur les relations de races (Chapoulie, 1998), Frazier, dont les penchants politiques étaient moins conservateurs ${ }^{25}$, introduisit des raisonnements en terme de relations de classe dans certains articles publiés dans des revues destinées à un public large au cours de la période qui précède son rattachement professionnel à la sociologie ${ }^{26}$. Un peu plus tard, pour mettre en évidence la différenciation interne de la population Noire, Frazier fut l'un des premiers à élaborer un classement par groupes de métiers de la population qui lui permit, à partir des recensements de population, de montrer l'inégale différenciation selon ce critère de la population des grandes villes, et d'étudier la répartition spatiale de ces groupes de métiers dans la ville de Chicago (Frazier, 1930). La construction d'un instrument permettant la vérification d'une des hypothèses de Burgess reflète aussi évidemment l'évolution interne des sciences sociales à cette époque vers un emploi plus systématique des techniques statistiques.

Dans les années suivantes, les analyses de Frazier accordent une place croissante à la prise en compte de la structure de classe de la communauté Noire. Plusieurs essais de Frazier proposent des analyses du jeu complexe entre les relations de races, la structure de classe et les effets de l'organisation sociale des communautés Noire et Blanche. Ainsi, pour ne donner qu'un exemple, Frazier avance que les Noirs, employés dans certaines institutions à côté de Blancs de classes moyennes, et qui ont un mode de vie de classe supérieure (en raison de leur position dans leur propres communautés et des standards liés à cette position dans la société américaine), tendent de ce fait à rester isolés des Blancs à côté desquels ils travaillent, qui ont eux-mêmes un mode de vie et des comportements de classe moyenne (Frazier, 1949). Si l'on reconnaît une idée avancée par Park - les relations personnelles et intimes sont le "grand solvant moral » qui mine les barrières de races (Park, 1926) - on voit la complexité supplémentaire que Frazier apporte dans sa mise en œuvre.

24 Une autre contribution de Frazier passe sans doute un peu inaperçue aujourd'hui. Une partie de ses efforts au cours de ses dernières années fut consacrée à distinguer la diversité des modes de relations possibles entre groupes de races ou, si l'on préfère, à préciser le sens du terme assimilation. L'exemple des Noirs américains le conduisit ainsi à distinguer entre l'intégration à la vie économique qui, selon ses observations, connaît des évolutions substantielles pendant et après la guerre de 1939-1945, 
l'acculturation (processus selon lequel la culture est adoptée par un groupe ethnique), l'assimilation (qui suppose une complète identification à un groupe) et le mélange des races par mariage (" amalgamation»). Contrairement à l'interprétation qui est souvent donnée de ses analyses, le dernier essai (posthume) de Frazier montre combien celui-ci resta jusqu'à la fin de sa carrière profondément incertain quant au devenir des Noirs américains, et donc loin d'admettre une théorie du cycle des relations entre les races (Frazier, 1962). Cet essai, destiné au congrès mondial de sociologie de Washington en 1962, y fut lu après la mort de l'auteur par Franklin Edwards.

Frazier y insiste judicieusement (comme la suite devait le démontrer) sur l'importance de l'émergence d'une classe moyenne dans les villes. Concluant sur la situation des Noirs des ghettos où nombre d'entre eux sont «non employés » et « non employables », et sur les compensations fréquemment recherchées par ceux-ci dans les drogues, les sectes religieuses ou nationalistes, Frazier remarque que «nul ne peut prédire l'aboutissement avec certitude de la rude épreuve de la civilisation américaine à laquelle ont été soumis les Noirs dans les villes » (Frazier, 1962 : 139).

Si Frazier partage avec Park l'exemple central de sa réflexion sur les relations entre les races - le cas des Noirs des États-Unis - celui qui apparait souvent aujourd'hui comme l'héritier le plus direct de Park, Everett Hughes, se pencha au contraire sur un cas singulier, marginal par rapport aux exemples historiques au centre des recherches américaines sur les relations entre races et cultures: les relations entre les groupes francophones et anglophones au Canada.

27 L'analyse de cet exemple, que poursuivit Hughes jusqu'à la fin de sa carrière, commença à la fin des années trente par l'étude d'une petite ville du Québec, où s'implantaient des industries relevant d'entreprises issues du Canada anglophone (Hughes, 1943). Comme il l'a lui-même raconté, Hughes abandonna rapidement le modèle d'une évolution inéluctable vers l'assimilation auquel ne semblait pas se conformer l'évolution des relations entre Canadiens français et anglais ${ }^{27}$. Cet exemple suggérait au contraire l'importance éventuelle de l'action des groupes ethniques sur eux-mêmes et leur propre contribution à la définition de leur avenir.

Un peu plus tard, revenu à Chicago, Hughes s'engagea dans des recherches sur ce qui était alors un problème d'actualité aux États-Unis : l'intégration des travailleurs Noirs dans la main-d'œuvre de la grande industrie, notamment au niveau des emplois semi qualifiés. Hughes réalisa plusieurs enquêtes à caractère ethnographique sur différentes usines pour comprendre les difficultés de l'introduction dans la main-d'œuvre de ces "étranges créatures ", comme l'écrit une fois Hughes avec ironie, que sont les femmes et les Noirs ${ }^{28}$. Ces recherches intègrent l'apport des analyses réalisées dans l'entourage de Warner, notamment celles de la Hawthorne, et sont attentives à des phénomènes jusque-là laissés de côté, comme les effets du contexte extérieur à l'usine, ou dans l'usine, le rôle des contremaîtres et celui du syndicalisme dans les réactions des différents types de travailleurs à l'entrée de nouveaux venus. Même si le petit nombre et la brièveté des comptes rendus publiés à l'issue de ces recherches ne permettent pas de mesurer l'apport d'une approche ethnographique aussi bien que ne le permettent d'autres travaux de sociologie du travail inspirés par Hughes, ils indiquent l'une des directions dans laquelle conduisait le cadre analytique de Park : l'étude minutieuse sur une base ethnographique de la division ethnique du travail dans les ateliers, les usines, les institutions ou les professions. Des essais de synthèse de Hughes tirent les 
conclusions de ces travaux et suggèrent de nouvelles pistes de recherche (Hughes, 1945 ; Hughes et McGill Hughes, 1952).

Conclusion

29 En proposant cette vue d'ensemble de la perspective de Park pour l'étude des contacts de races et de cultures, j'ai d'abord montré que celle-ci ne se réduisait pas à quelques propositions « de fait » que l'histoire ou le «progrès » des sciences sociales rendraient maintenant obsolètes. J'ai soutenu qu'il s'agissait d'un cadre de référence souple défini par des catégories d'analyse et par une liste de questions. J'ai suggéré que les ajouts et réaménagements auxquels ont contribué les successeurs de Park lorsqu'ils ont été confrontés à des phénomènes nouveaux ou précédemment négligés, n'ont pas été indépendants des évolutions internes des sciences sociales, notamment de la systématisation des méthodes de documentation à partir des années trente, ou des changements de contexte socio-politique, comme ceux qui conduisirent à accorder davantage d'attention à des phénomènes structurels comme les relations de classe. $\mathrm{Si}$ l'on revient à la caractérisation que j'avais avancée au début - le cadre de référence de la sociologie des relations de races et cultures de Park comme abstraction, à partir du point de vue du groupe politiquement hégémonique, de l'expérience historique des États-Unis de l'époque -, on peut avancer que le développement des recherches a conduit à prendre en compte certains des phénomènes que ce point de vue rendait initialement aveugle.

La plupart des travaux que l'on trouve sous le label de la tradition de Chicago partagent le souci de lier l'analyse des phénomènes les plus concrets qui ne peuvent être saisis que par une démarche ethnographique et celle des phénomènes globaux qui caractérisent les sociétés contemporaines - ce que Park désignait par référence à son expérience du journalisme, comme les «Big News». Mais au-delà de cette caractéristique commune, les différentes recherches ne forment certainement pas un ensemble unifié dont on pourrait extraire une théorie organisée (mais on sait aussi que celles-ci ne résistent jamais bien au temps dans notre discipline). Il s'agit plutôt d'un ensemble composé de recherches de terrain menées dans le cadre légué par les prédécesseurs, utilisé sans respect particulier pour leurs propositions substantielles, et d'essais qui proposent des généralisations qui n'ont été que très partiellement soumises à l'épreuve de la vérification empirique. Dans ce domaine de recherche comme dans les autres, le caractère composite des travaux mis sous ce label constitue à la fois la faiblesse et la force de la tradition de Chicago.

31 La contribution de la tradition de Chicago à l'étude des contacts de race et de culture semble assez typique de ce que sont, dans les meilleurs des cas, les apports de ce genre d'entreprises collectives à un domaine de recherche. Une partie du cadre de référence proposé par Park est passé insensiblement dans la culture commune des chercheurs de ce domaine aux États-Unis et se retrouve donc dans les principes de base des études actuelles: l'accent sur les relations et non sur les groupes substantiels, le souci comparatiste ne sont ici que des exemples. Une autre partie, à peu près totalement oubliée, est cependant susceptible de fournir des idées pour des recherches de terrain, et d'être ainsi réintroduite à l'occasion comme "nouvelle », comme d'autres l'ont déjà remarqué (Lal, 1983). 


\section{BIBLIOGRAPHIE}

ADORNO Theodore, W., FRENKEL-BRUNSWICK Else, LEVINSON Daniel and SANFORD R. (1950) The Authoritarian Personality, New York, Harper and Brothers.

BAHR Howard M., JOHNSON Theodore J. and SEITZ Ray M. (1971) « Influential Scholars and Works in the Sociology of Race and Minority Relations, 1944-1968 », The American Sociologist, 6 (4), pp. 296-298.

BLASSINGAME John W. (1972) The Slave Community. Plantation Life in the Antebellum South, New York, Oxford University Press.

CHICAGO COMMISSION OF RACE RELATIONS (1922) The Negro in Chicago. A Study of Race Relations and a Race Riot in 1919, Chicago, University of Chicago Press.

DU BOIS William, BURGHARDT E. (1899) The Philadelphia Negro : A Social Study, Philadelphia, The University of Pennsylvania Press.

FRAZIER E. Franklin (1925) « Durham : Capital of the Black Middle Class », in Alain Locke (ed) : The New Negro, New York, A. and C. Boni, pp. 333-340.

FRAZIER E. Franklin (1928) « La Bourgeoisie Noire », Modern Quarterly, 5 (November) ; also in V. F. Culverton (ed) : Anthology of the Negro Literature in America, New York, The Modern Library, 1930 : 329-388.

FRAZIER E. Franklin (1932) The Negro Family in Chicago, Chicago University of Chicago Press.

FRAZIER E. Franklin (1939) The Negro Family in the United States, Chicago University of Chicago Press.

FRAZIER E. Franklin (1940) Negro Youth at the Crossways : Their Personality Development in the Middle States, Washington, (DC) American Council of Education.

FRAZIER E. Franklin (1949 a) The Negro in the United States, New York, MacMillan.

FRAZIER E. Franklin (1949 b) « Race Contacts and the Social Structure », American Sociological Review, 14 (1), pp. 1-11.

FRAZIER E. Franklin (1953) « Theoretical Structure of Sociology and Sociological Research », British Journal of Sociology, 4 (December), pp. 292-311.

FRAZIER E. Franklin (1955) Bourgeoisie Noire, Paris, Plon.

FRAZIER E. Franklin (1957) Race and Culture Contacts in the Modern World, New York, A Knopf.

FRAZIER E. Franklin (1962) « Conditions of Negroes in American Cities », Transactions of the Fifth World Congress of Sociology, Washington, DC, Vol III, pp. 133-139.

FRAZIER E. Franklin (1964) The Negro Church in America, New York, Schoken (Liverpool University Studies in sociology).

GILKESON John S. (1995) « American Social Scientists and the Domestication of "Class" 1929-1955 », Journal of The History of The Behavorial Sciences, 31 (July), pp. 331-346.

GUTMAN Herbert G. (1976) The Black Family in Slavery and Freedom, Oxford, Basic Blackwell.

HERSKOVITS Melville J. (1941) The Myth of the Negro Past, New York, Harper and Brothers.Trad. F. L'Héritage noir : mythe et réalité, Paris, Présence africaine, 1966. 
HUGHES Everett C. (1943) French Canada in Transition, Chicago, University of Chicago Press. (Traduction française par Jean-Charles Falardeau : Rencontre de deux mondes. La Crise d'industrialisation du Canada Français, Montreal, Parizeau, 1945 ; réédition en 1972 par Les Éditions du Boreal Express, Montreal).

HUGHES Everett C. (1945) «Dilemmas and Contradictions of Status », American Journal of Sociology, 50 (5) : 353-59. (Traduction française in Le Regard sociologique, Paris 1995, Éditions de l'EHESS, pp. 187-197).

HUGHES Everett C. (1946 a) « The Knitting of Racial Groups in Industry », American Sociological Review, 11 (5) : 512-19. (Traduction française in Le Regard sociologique, Paris 1995, Éditions de l'EHESS : 251-263).

HUGHES Everett C. (1946 b) « Race Relations in Industry », in W. F. Whyte (ed.) : Industry and Society. New York, McGraw-Hill, 1946, pp. 107-122.

HUGHES Everett C. (1963 a) « The Natural History of a Research Project : French Canada », Anthropologica, 5 (2), in Hughes, 1971, pp. 530- 542.

HUGHES Everett C. (1963 b) « Race Relations and the Sociological Imagination », American Sociological Review, 28 (6): in Hughes, 1971, pp. 478-495.

HUGHES Everett C. (1971) The Sociological Eye. Selected Papers, Chicago, Aldine.

HUGHES Everett C., MACGILL HUGHES Helen (1952) Where Peoples Meet : Racial and Ethnic Frontiers, Glencoe, The Free Press.

HUGHES Everett C., HILL, MOZELL C. (1946) « The Negro Man and his Work. Social Differenciation among the Negro Male Worker », The Southwest Journal, 1946, 2 (2), p. 129-139.

HUGHES Everett C., HILL, MOZELL C. (1985) Hull House Maps and Papers, by Residents of Hull House, a Social Settlement. A Presentation of Nationalities and Wages in a Congested District of Chicago, Together with Comments and Essays on Problem Growing out of the Social Conditions, New York, Crowell.

KARL Barry D. (1983) The Uneasy State. The United States from 1915 to 1945, Chicago, University of Chicago Press.

LAL Barbara Ballis (1983) « Perspectives on Ethnicity : Old Wine in New Bottles », Ethnic and Racial Studies, 6 (2), pp. 154-173.

LAL Barbara Ballis (1990) The Romance of Culture in an Urban Civilization. Robert E. Park on Race and Ethnic Relations in Cities, London, Routledge.

LYMAN Stanford M. (1972) The Black American in Sociological Thought, New York, Putnam.

LYMAN Stanford M. (ed.) (1992) Militarism, Imperialism and Racial Accomodation. An Analysis and Interpretations of the Early Writings of Robert E. Park, Fayetville, The University of Arkansas Press.

MCKEE James B. (1993) Sociology and the Race Problem. The Failure of Perspective, Urbana (Ill.), University of Illinois Press.

MATTHEWS Fred H. (1977) Quest for an American Sociology : Robert E. Park and the Chicago School, Montréal Mc Gill-Queen's University Press.

MYRDAL Gunnar (1944) An American Dilemma : The Negro Problem and Modern Democracy, New York, Harper and Row.

PARK Robert E. (1913) « Negro Home Life and Standards of Living », Annals of the American Academy of Political and Social Science, 49 (September), pp. 147-163, in Lyman 1992, pp. 262-275. 
PARK Robert E. (1922) The Immigrant Press and Its Control, New York, Harper.

PARK Robert E. (1926) « Our Racial Frontier on the Pacific », Survey Graphic, LVI (May), pp. 192-96 (in Race and Culture, Glencoe, Illinois, The Free Press, 1950, pp. 150).

PARK Robert E. (1930 b) « Social Assimilation », International Encyclopaedia of Social Sciences, vol 2, pp. 281-283.

PARK Robert E. (1939 b) « The Nature of Race Relations », in Edgar T. Thompson (ed), Race Relations and the Race Problem, Durham, Duke University Press, pp. 3-45, in Race and Culture.

PARK Robert E. (1950-1955) The Collected Papers of Robert Ezra Park, ed by Everett C Hughes, Charles S. Johnson, Jitsuichi Masuaoka, Robert Redfield, Louis Wirth, Glencoe (Ill) : Free Press; Vol 1, Race and Culture, 1950; Vol II, Human Communities : The City and Human Ecology, 1952 ; Vol III, Society: Collective Behavior, News and Opinion, Sociology and Modern Society, 1955.

PARK Robert E., BURGESS Ernest (1921) Introduction to the Science of Sociology, Chicago, University of Chicago Press.

PARK Robert E., MILLER Herbert A (1921) Old World Traits Transplanted, New York, Harper.

PLATT Anthony M. (1991) E. Franklin Frazier Reconsidered, New Brunswick, Rutgers University Press.

RAUSHENBUSH Winifred (1979) Robert Park. Biography of a Sociologist, Durham, Duke University Press.

SCHNAPPER Dominique (1998) La relation à l'autre, Paris, Gallimard.

SINGER L. (1962) «Ethnogenesis and Negro-Americans Today », Social Research, 29 (4), pp. 419-432.

STEINBERG Stephen (1981) The Ethnic Myth. Race Ethnicity, and Class in America, Boston, Beacon Press.

\section{NOTES}

1. -La contribution de Thomas à ce domaine d'étude a été ici laissée de côté de manière un peu arbitraire, mais aussi parce que l'influence de Thomas sur les recherches empiriques des années postérieures est passée en partie par l'interprétation qu'en donne Park. Les formulations de Park sont d'ailleurs plus générales et plus facilement utilisables dans des recherches sur des terrains divers que celles de Thomas.

2. -Cet article s'appuie sur un ensemble de recherches sur l'histoire de la sociologie aux États-Unis, qui repose notamment sur le dépouillement des archives de Park, Burgess et Hughes déposées à la Joseph Regenstein Library de l'Université de Chicago.

3. -E.C. Hughes : Race and Language, Lecture Florida State, 19 janvier 1967 in J. Regenstein Library, Université de Chicago, Archives E.C. Hughes, carton 109, dossier 16. 4. -Ces points sont abondamment mis en évidence dans le rapport de la Chicago Commission of Race Relations (1922).

5. -L'idée de traduire le terme qui désigne le second de ces processus, « accommodation » par le terme français identique semble bizarrement se répandre actuellement en France et donne un air mystérieux au compromis (Il s'agit probablement d'une dérivation malheureuse d'un sens rare relevé par le dictionnaire Robert : accommodation : action d'accommoder ou de s'accommoder). 
6. -Park reconnaît d'ailleurs clairement l'imprécision du terme dans un essai rarement cité, postérieur à la publication de l'Introduction ot the Science of Sociology : l'article «Assimilation » de l'Encyclopaedia (Park, 1930 b).

7. -Par exemple, l'ouvrage classique de Steinberg, 1981 (qui a par ailleurs d'autres mérites) réduit Park au rôle de théoricien du Melting Pot. Steinberg oublie d'ailleurs significativement l'un des processus distingués par Park. Le caractère approximatif des lectures de Park (que relevait déjà Hughes dans les années cinquante) a été sans doute favorisé par le style de ses essais - reposant souvent sur de libres associations d'idées - et par les lectures non historiquement contextualisées, ainsi que par l'organisation thématique, et non chronologique, du principal recueil, Race and Culture (1950). La réduction de la sociologie de Park à ce cycle se retrouve dans des présentations françaises récentes : voir par exemple Schnapper, 1998 : 194-195. 8. -Il est facile de montrer qu'il en va de même pour Blumer et Wirth.

9. -Robert Park, manuscrit non daté [entre 1930 et 1944], in archives Park, Joseph Regenstein Library, carton 5, dossier 2. L'ouvrage de Lyman (1972) propose l'analyse à ma connaissance la plus détaillée de l'idée de cycle de relations entre les races de Park. Ma divergence avec cette interprétation, qui s'appuie sur une vue d'ensemble de la sociologie de Park (à l'inverse de la plupart de celles qui sont en général derrière l'invocation de ce cycle) tient à ce que je ne crois pas qu'on parvienne à une bonne appréciation de la perspective de Park en couchant ses essais sur le lit de Procuste de la philosophie. Je ne suis évidemment ni le seul ni le premier à critiquer la réduction de la sociologie des relations entre les races de Park à ce cycle : voir Lal, 1990 ; McKee, 1993 : 110-111 pour des exemples récents.

10. -L'attention à cet aspect apparaît pour la première fois dans un article de Singer (1962) qui n'eut d'écho qu'un peu plus tard.

11. -Après la publication de l'ouvrage de Myrdal, en 1944, ce fut pour une longue période sur le fatalisme de Park - sa conviction que les changements des dispositions législatives n'étaient pas susceptibles de modifier le cours des relations entre les races - que se focalisèrent les critiques.

12. -Je n'ignore évidemment pas qu'on trouve chez Park dans un article de 1913 une formulation malheureuse, et souvent relevée plus tard, qui évoque le « tempérament racial » des Noirs. Voir sur ce point Matthews, $1977: 172$.

13. -Ce point est souligné dans une lettre envoyée à Horace Cayton en 1943 : « Si des conflits se produisent comme conséquence de leurs efforts [celui des Noirs] pour obtenir leur place, ce sera parce que les Blancs en prennent l'initiative. De tels conflits se produiront probablement et sont plus ou moins inévitables mais leur situation sera ensuite meilleure ", cité in Matthews, $1977: 189$. Matthews cite également une autre lettre à son ancienne assistante Winifred Raushenbush où Park ne se déclare l'adversaire des émeutes que si les Noirs doivent toujours perdre.

14. - Ces recherches constituent pour partie la postérité intellectuelle des ouvrages de Myrdal, 1944 et d'Adorno et al., 1950.

15. -Les Iles Hawaï pour Park fournissent encore plus que la Côte Ouest l'exemple du mélange des populations par intermariage.

16. -Comme le remarque Hughes dans une lettre à James Short, Park n'a pas fait autre chose qu' « en visiter " (Archives Hughes, carton 55, dossier 19).

17. -La contribution de Park à cette recherche est contestée : l'ouvrage est maintenant parfois attribué au seul W.I. Thomas (qui, en 1921, était interdit de signature par la fondation Carnegie en raison des conditions de son éviction de l'Université de Chicago). 
S'il ne fait aucun doute que la contribution d'Herbert Miller fut très limitée, il n'est pas sûr qu'il en aille de même pour celle de Park. Le témoignage de Winifred Raushenbush, alors l'assistante de Park et qui travailla avec Park et Thomas sur le livre (in Archives Park, addenda, dossier 9) insiste au contraire sur l'implication directe de Park dans cette recherche. Vers 1975, Hughes semblait juger cependant impossible de mettre en avant celle-ci dans le contexte de l'époque (qui avait vu la « découverte » de la contribution de Thomas à l'ouvrage) : voir Raushenbush, 1979 : 86-94.

18. -Un terme prématuré fut mis à cette recherche collective par son mécène, l'Institute of Social and Religious Research ; un compte rendu très partiel fut publié dans un numéro spécial du Survey Graphic de 1926.

19. -Voir notamment quelques-uns des articles récemment publiés dans le recueil de Lyman, notamment « Negro Home Life and Standards of Living », 1913 in Lyman, 1992 :

262-275. L'importance de l'expérience de l'univers des Noirs du sud est soulignée dans une lettre de la fille de Park, Margaret Park-Redfield, qui avait été elle même formée comme ethnologue (in Archives Matthews, Joseph Regenstein Library Chicago).

20. -Une évaluation de l'influence apparente dans ce domaine des différents auteurs et ouvrages se trouve dans l'article de Bahr, Johnson et Seitz, 1971.

21. -Rappelons que Frazier (1897-1962), qui appartient à la génération de ceux qui ont accompagné la mise en place de l'organisation qui est encore aujourd'hui celle de la sociologie américaine, est le premier Noir élu président de l'Association américaine de sociologie, en 1948.

22. -Depuis les années soixante, différentes recherches historiques ont conduit à l'abandon d'une partie des hypothèses à peu près unanimement admises jusque-là sur l'absence d'héritage africain chez les Afro-américains : voir Blassingame (1972), Gutmann (1976). Cette évolution concernant les faits considérés comme acquis n'implique évidemment pas que les analyses de Frazier ne contiennent pas d'idées fécondes pour de nouvelles recherches. Une biographie récente de Frazier (Platt, 1991) attribue une partie de l'oubli dont a souffert l'œuvre de Frazier ces trente dernières années à la réinterprétation de ses analyses par N. Glazer et P. Moynihan qui inspirèrent une politique fédérale en matière de relations entre les races qui n'apparaît pas rétrospectivement comme un grand succès.

23. -Le compte rendu de cette recherche a été publié après la mort de Frazier, en 1964. Je laisse ici de côté un des livres les plus diffusés de Frazier (1949a), qui constitue plutôt une synthèse d'ensemble qu'une analyse reposant sur un matériel de première main.

24. -Cet essai, très réservé à l'égard de Warner et des recherches sur les attitudes inspirées de Myrdal qui étaient à la mode à l'époque, n'explicite aucune critique à l'égard de Park. Frazier reproche cependant implicitement à Park une insuffisante attention aux actions à dimension politico-économique de la communauté Blanche expressément destinées à contrôler la communauté Noire, notamment en matière d'habitat, d'emploi, etc.

25. -Frazier avait notamment dans les années vingt collaboré à la revue Messenger fondée par le journaliste et leader syndical A. Philip Randolph qu'on peut qualifier de marxiste indépendant (sur les convictions politico-sociales de Frazier voir Platt, 1991). 26. -Frazier, rappelons-le, a enseigné différentes matières, puis a été professeur de travail social à Atlanta avant d'entreprendre une thèse en sociologie à l'Université de Chicago.

27. -Selon son témoignage, Hughes arriva à Montréal avec deux hypothèses concernant les canadiens francophones, qui découlaient de sa formation à l'Université de Chicago : 
$1^{\circ}$ ) les francophones étaient un groupe d'immigrants en cours d'assimilation; $2^{\circ}$ ) ils constituaient une minorité nationale. Hughes renonça rapidement à la première affirmation, à laquelle adhéraient « spontanément » certains de ses étudiants (des anglophones). À travers ses lectures, il découvrit que les francophones n'étaient pas une minorité revendiquant le rattachement à la France ou leur autonomie politique : ils revendiquaient avant tout l'autonomie culturelle et l'égalité avec les anglophones. La culture anglophone ne leur apparaissait pas supérieure à la leur et rien n'indiquait qu'ils adhéraient à l'idée qu'une culture nationale uniforme était souhaitable ou inévitable.

28. -Des comptes rendus partiels de ces recherches furent publiés - Hughes, 1946 a ; 1946 b ; Hughes, Hill, 1946 - mais non l'ouvrage final que Hughes envisageait d'écrire, dont on trouve cependant un plan et des esquisses dans les archives de Hughes à l'Université de Chicago : voir les cartons 98 et 99. Dans le carton 98, dossier 9, se trouve notamment un « Plan for race in industry book ».

\section{RÉSUMÉS}

L'étude des relations entre les races, prises au sens culturel, est un aspect longtemps négligé de la tradition sociologique de Chicago entre 1910 et 1960. Pourtant Park, dès le début du Xx siècle, les étudie comme des relations réciproques entre groupes ethniques majoritaires et minoritaires. Cet article insiste sur le caractère hypothétique, pour Park, de sa théorie du cycle de relation entre migrants et descendants des fondateurs des États-Unis. Il donne cinq arguments qui vont dans ce sens. Le caractère hypothétique est justifié par les travaux de deux des plus proches élèves de Park : E. Franklin Frazier qui montra la difficile entrée des Noirs américains dans une complète assimilation, même lorsqu'ils appartiennent à la petite bourgeoisie, difficulté dont témoigne la rareté des mariages avec des Blancs ; et E.C. Hughes qui dut abandonner son hypothèse de l'assimilation des Canadiens francophones à la culture anglo-saxonne et envisager, comme futur probable du Canada, une culture métissée.

The Chicago Tradition and the Study of Race Relations. -- The study of race relations, in the cultural meaning of the term, is a long-neglected aspect of the Chicago sociological tradition between 1910 and 1960. Yet since the beginning of the twentieth century, Park had studied them as reciprocal relations between majority and minority groups. This article emphasizes the hypothetical nature of Park's theory of the cycle of relations between migrants and the descendants of the founders of the United States. He presents five propositions to this effect. Their hypothetical character is borne out by the work of two of the students who were closest to Park. E. Franklin Frazier shows the difficulty of Black Americans to fully assimilate, even when they belong to the petty bourgeoisie, as indicated by their few marriages with Whites. E. C. Hughes was obliged to abandon his hypothesis regarding the assimilation of French Canadians to Anglo-Saxon culture was led to propose a hybrid culture as the likely future for Canada.

La tradicion de Chicago el estudio de las relaciones entre las razas .-- El estudio de las relaciones entre las razas, en el sentido cultural del término, fue un aspecto que la tradicion sociológica de Chicago descuidó durante un largo periodo entre 1910 y 1960. Sin embargo, Park, desde el inicio del siglo XX, las estudia como relaciones reciprocas entre grupos étnicos mayoritarios y 
minoritarios. Este articulo insiste sobre el carácter hipotético que tenía para Park su teoría del ciclo de relacion entre inmigrantes y descendientes de los fundadores de los Estados Unidos. El autor propone en ese sentido, cinco argumentos. El carácter hipotético de los escritos de Park fue justificado por los trabajos de dos de sus estudiantes. E. Franklin Frazier, puso en evidencia la dificultades de los negros americanos para realizar una asimilación exitosa, aun en casos de pertenencia a la pequeña burguesía. De ello rinde cuenta precisamente la rareza de matrimonios con blancos. El otro estudiante es E.C. Hughes, quien debió abandonar su hipótesis de una asimilación de los canadienses franco parlantes frente a la cultura anglosajona para proponer, como el futuro mas probable para Canadá, la elaboración de una cultura mestiza.

INDEX

Mots-clés : concept, école de Chicago, recherche, relations inter-raciales, ville

\section{AUTEUR}

\section{JEAN-MICHEL CHAPOULIE}

Professeur de Sociologie, Université de Paris-Sorbonne, Directeur du centre de Recherches sociologiques et historiques sur l'Éducation. 